\title{
Téoros
}

Revue de recherche en tourisme

\section{Vacances des femmes}

\section{Temps de loisir?}

\section{Nicole Samuel}

Volume 16, numéro 3, automne 1997

Femmes et tourisme

URI : https://id.erudit.org/iderudit/1073310ar

DOI : https://doi.org/10.7202/1073310ar

Aller au sommaire du numéro

Éditeur(s)

Université du Québec à Montréal

ISSN

0712-8657 (imprimé)

1923-2705 (numérique)

Découvrir la revue

\section{Citer cet article}

Samuel, N. (1997). Vacances des femmes : temps de loisir ? Téoros, 16(3), 4-8.

https://doi.org/10.7202/1073310ar

Ce document est protégé par la loi sur le droit d'auteur. L'utilisation des services d'Érudit (y compris la reproduction) est assujettie à sa politique d'utilisation que vous pouvez consulter en ligne.

https://apropos.erudit.org/fr/usagers/politique-dutilisation/
Cet article est diffusé et préservé par Érudit.

Érudit est un consortium interuniversitaire sans but lucratif composé de l’Université de Montréal, l'Université Laval et l'Université du Québec à Montréal. Il a pour mission la promotion et la valorisation de la recherche. https://www.erudit.org/fr/ 


\section{VACANCES DES FEMMES}

TEMPS DE LOISIR?

\section{Nicole Samuel, sociologue CNRS, Paris}

En 1970, Evelyne Sullerot, sociologue francaise auteure de nombreux ouvrages sur la condition féminine, écrivait: "Quant aux vacances, elles sont pour beaucoup de femmes un rêve décevant. D'abord parce qué la mottié des Français (NDLR: aujourd'hui, en $1997,23,5 \%)$ ne partent jamais en vacances... Ensuite parce que pour l'autre moitie, pour ceux quil wont en vacances, les femmes ne sont dans la majorite des cas que des ménagères en déplacement. Au mieux, si la famille descend à l'hôtel, elles sont des mères qui s'occupent de leurs enfants ailleurs qu'au domicile habifuel. \& (Sullerot, 1970: 4)

Qu'en est-il aujourd'hui, après plus d'un quart de siècle marqué par d'importants changements sociaux? Les vacances ontelles cessé d'être pour les femmes un rêve décevant? Correspondent-elles maintenant pour elles à un temps de loisir?

Il importe de souligner que le concept de vacances fait appel à deux notions: d'une part le congé qui désigne le temps libéré des occupations de travail professionnel et qui $n$ 'implique pas forcément l'idée de départ; et d'autre part le tourisme, défini part l'INSEE comme un déplacement hors de la résidence principale, pour une durée d'au moins 24 heures et inférieure à quatre mois, pour l'un des motifs suivants: agrément, santé, mission, voyage d'affaires, voyage scolaire (Rauch, 1993: 7-8). Les vacances peuvent alors se définir comme une période de temps libéré du travail professionnel pendant laquelle s'affirme le droit au loisir et comportant ou non un départ pour un voyage d'agrément.

Quant au loisir, on le définira comme le seul contenu du temps orienté vers la réalisation de la personne comme fin dernière (Dumazedier, 1974:93) tout en soulignant que le loisir des femmes étant caractérisé par son interpénétration avec les autres sphères de la vie et n'étant pas toujours sếparé des obligations, le concept de loisir féminin a été étendu par la recherche féministe à l'expérience du bien-être, de la relaxation, de la détente, du plaisir. Une attention particulière est alors donnée aux significations et aux relations liées au loisir, considéré comme une interaction vécue dans un contexte, caractérisé par un choix, une motivation intrinsèque et un sentiment de plaisir et de satisfaction (Henderson, 1991: 1; Samuel, 1996; 6). De telles caractéristiques se retrouvent-elles pour les femmes dans leur temps de vacances?

\section{D'IMPORTANTS CHANGEMENTS SOCIAUX DANS LA PÉRIODE RÉCENTE}

Évolution du statut des femmes et répercussions sur leur temps de loisir

Au cours des dernières décennies, le statut des femmes et leur image sociale se sont modifiés (Samuel, 1996: 1). Leur accès à la formation et à l'éducation à tous les niveaux a augmenté avec une hausse correspondante de leurs qualifications. II y a eu d'indéniables progrès dans leur accès au marché du travail et, comme tous les salariés, les femmes actives ont droit à des congés payés par leur employeur. Mais partout dans le monde, même si c'est à des degrés divers, ce sont les femmes (actives ou non) qui continuent à assumer la plus grande partie des tâches domestiques et du soin des enfants (UN, 1991: 82). Or ces tâches ne connaissent jamais de rếpit. Il s'ensuit que la distribution du temps entre les sexes reste inégale pratiquement partout dans le monde et que 1'accès des femmes au loisir et aux vacances demeure difficile. Les études de budgets-temps conduites dans de nombreux pays (UN, 1991: 103-104) montrent cette inégalité, les hommes ayant généralement plus de temps libre que les femmes. Le tableau 1 résume cette situation:

On remarque une seule exception: celle des Pays-Bas, et encore seulement lorsqu'il s'agit des actifs a plein temps. Cette remarque en appelle une autre: sur l'ensemble des pays cités, l'écart entre les deux sexes est moins grand dans cette catégorie et il augmente dans la plupart des cas à mesure qu'on va vers les inactifs, la pratique du temps partiel se trouvant en général à mi-chemin. Ceci peut être interprété comme un signe de changement apporté à leur temps de loisir par l'entrée des femmes sur le marché du travail, les actives à plein temps se trouvant (paradoxalement si l'on pense à leur double journée de travail!) privilégiées par rapport aux actives à temps pertiel et aux inactives en ce qui concerne la distribution du temps libre et se rapprochant ainsi du mode de vie et de leurs collègues masculins. Mais 
TABLEAU 1

Temps libre dans sept pays (en heures par semaine)

\begin{tabular}{|l|c|c|c|c|c|c|c|}
\hline & $\begin{array}{c}\text { ROYAUME UNI } \\
\mathbf{1 9 8 4}\end{array}$ & $\begin{array}{c}\text { CANADA } \\
\mathbf{1 9 8 1}\end{array}$ & $\begin{array}{c}\text { DANEMARK } \\
\mathbf{1 9 7 5}\end{array}$ & $\begin{array}{c}\text { FRANCE } \\
1986\end{array}$ & $\begin{array}{c}\text { NORVĖGE } \\
1981\end{array}$ & $\begin{array}{c}\text { PAYS-BAS } \\
\mathbf{1 9 8 0}\end{array}$ & $\begin{array}{c}\text { USA } \\
1985\end{array}$ \\
\hline $\begin{array}{l}\text { Hommes actifs } \\
\text { à temps plein }\end{array}$ & 38,1 & 40,3 & 46,2 & 35,6 & 39,8 & 46,3 & 35,4 \\
\hline $\begin{array}{l}\text { Femmes actives } \\
\text { à temps plein }\end{array}$ & 35,1 & 36,8 & 43,2 & 27,7 & 37,6 & 49,4 & 33,6 \\
\hline $\begin{array}{l}\text { Hommes actifs } \\
\text { à temps partiel }\end{array}$ & 48,3 & 56,0 & 55,1 & 47,3 & 55,4 & 70,8 & 55,5 \\
\hline $\begin{array}{l}\text { Femmes actives } \\
\text { à temps partiel }\end{array}$ & 36,0 & 40,0 & 45,5 & 32,1 & 41,1 & 56,6 & 40,4 \\
\hline Hommes inactifs & 63,2 & 69,7 & 67,4 & 62,8 & 68,3 & 66,4 & 57,6 \\
\hline Femmes inactives & 49,3 & 55,9 & 56,8 & 47,5 & 51,6 & 48,0 & 48,9 \\
\hline
\end{tabular}

Source: Roy, 1987

on ne dispose malheureusement pas de données permettant de savoir comment se répercute cette observation sur le temps de vacances des différentes catégories de femmes.

\section{DÉVELOPPEMENT DES CONGÉS PAYÉS}

Par ailleurs, au cours de ces mêmes dernières decennies, le droit aux congés payés et aux vacances n'a cessé de se renforcer. Avant 1905, aucune loi dans aucun pays ne garantissait un tel droit, même si la coutume en existait dans certains cas, et les congés payés ne se répandirent que progressivement: en 1936, ils avaient été légalisés dans une douzaine de pays européens et une convention internationale avait été proposée par le BIT pour coordonner leur politique dans ce domaine. Cela ouvrit la voie à un élargissement de cette mesure sociale aux soixante états et territoires qui ratifièrent la convention internationale ${ }^{\circ} 52$ de l'OIT (Organisation Internationale du Tourisme) sur les congés payés. Celle-ci prévoyait une durée minimale de six jours (Lanquar, 1994: 11). Après la deuxième guerre mondiale, I' article 24 de la Declaration universelle des droits de l'homme, adoptée le 10 décembre 1948 par les Nations Unies, spécifie que toute personne a droit à des congés payés. Le 24 juin 1970, l'année où Evelyne Sullerot écrivait le commentaire cité plus haut, une nouvelle convention $\left(\mathrm{n}^{\circ} 132\right)$ fut adoptée par l'OIT Elle prévoyait un mi= nimum de trois semaines de congés payés
(Lanquar, 1994: 11; Lanquar et Raynouard, 1995: 16; Richez et Strauss, 1995: 385). Enfin en 1982, le Document d'Acapulco sur le Tourisme mondial énonça un certain nombre de priorites: le droit au repos, aux loisirs, aux congés payés et la création de conditions légales et sociales rendant plus facile pour toutes les catégories sociales d'avoir des vacances (Lanquar, 1994: 20).

C'est ainsi qu'en 1985 entre 1500 et 1770 millions de personnes (en majorité des salariés et leurs familles) bénéficiaient de congés payés avec la répartition régionale suivante: en Afrique, de 75 à 150 millions; dans les Amériques, de 380 a 400 millions; en Asie et Pacifique, de 410 à 520 millions; en Europe, de 560 à 610 millions; au Moyen-Orient, de 75 à 90 millions, On voit que la marge d'évaluation est forte! De plus, tous les bénéficiaires de congés payés ne partent pas en vacances et beaucoup ne font pas de tourisme. En 1986, sur les 250 millions d'Européens âgés de plus de quinze ans, 110 millions n"étaient pas partis en vacances, dont $44 \%$ affirmaient qu'ils n'en avaient pas les moyens (Communauté Européenne, 1986). Pour sept pays seulement de la Communauté Européenne, le taux de départ dépassait $75 \%$ pour les plus de quinze ans. En France, il atteignait $76.5 \%$ en 1995 , ce qui correspondait a un nombre total de séjours représentant $93,6 \%$ de l'ensemble des voyages effectués (Mémento du Tourisme, 1996/1997: 92 et 80 ). Il faut d'ailleurs noter que souvent le départ n'occupe qu'une partie des congés: c'est ainsi qu'entre mai $1994 \mathrm{et}$ mai 1995, plus de la moitié des Français (54\%) ont passé plus de la moitié de leurs vacances chez eux (Sondage SOFRES, 1995).

Dans ce double contexte - évolution du statut des femmes et diffusion des congés payés et des vacances - généralement considéré comme positif mais marqué par d'importantes limitations, qu'en est-il des vacances des femmes? Constituent-elles un temps de loisir?

\section{PEU DE DONNÉES QUANTITATIVES SUR LES VACANCES DES FEMMES}

On est tout d'abord surpris de constater la rareté des données existant à ce sujet sur le plan quantitatif. En effet, les pratiques de vacances ne sont pas prises en compte dans les études du loisir culturel et sportif. D'autre part, les budgets-temps ne concernent en général que les emplois du temps de la vie quotidienne. Mais faisant apparaître combien le poids du travail domestique pèse sur le loisir des femmes, ces études conduisent à penser que. pendant les vacances, cette situation a peu de chance d'être profondément modifiée, puisque nombreuses sont les femmes qui les passent à la maison et rares sont celles qui, partant en vacances, logent à l'hôtel où elles sont ếvidemment déchargées du travail domestique quotidien. En France, les séjours de vacances à l'hôtel ne représentaient en 1995 que $14,2 \%$ de l'en- 
semble des séjours de vacances (Mémento du Tourisme. 1996/1997; 102). Différentes études montrent d"ailleurs que le travail domestique non rémunéré, surtout exécuté par les femmes, persiste pendant les vacances (Crawford et al., 1992) (Bittman, 1991 et Cunningham, 1994, cités par Davidson, 1996: 91), Quant aux analyses des départs en vacances, si elles apportent des informations sur le rôle de l'âge, de la catégorie socio-professionnelle et du lieu de résidence, elles ne s'intéressent pas au taux de départ respectif des hommes et des femmes. C'est le cas en France, mais aussi en Grande-Bretagne et au niveau de l'OMT (Deem, 1993:23). On parle des départs en vacances en termes géneraux comme s'ils concernaient indistinctement les hommes et les femmes. Certes, il est probable que parmi ces dernières, celles qui ont un partenaire et/ou des enfants partent en vacances avec eux, puisqu'un des buts fréquents des départs est d'améliorer les relations qu'on a avec ses proches (Tysoe, 1985, cité par Deem, 1996a: 19). En Grande-Bretagne, cela représentait, en 1992, 73,9\% du total des ménages (Deem, 1996: 19).

Mais on ignore ce qu'il en est pour les fermmes de tous âges célibataires ou vivant seules. De ci de là, on glane quelques informations dispersées. On apprend par exemple qu'au total les Européens sont plus nombreux à voyager que les Européennes (European Travel Commission Report, 1986). Il en est de même parmi les jeunes qui ont utilisé le programme "Interrail», commencé au début des années 70 et donnant droit à un nombre illimité de trajets sur le réseau des trains européens pour un prix avantageux: on y a répertorié $57 \%$ de garçons et $43 \%$ de filles. Mais une différence régionale apparaît: si les premiers représentent la majorité parmi les Marocains (96\%); les Turcs $(81 \%)$; les Grecs $(75 \%)$, les filles au contraire sont plus nombreuses dans le Nord: au Danemark ( $56 \%$ ), en Finlande $(54 \%)$ et en Suède $(53 \%)$ (Kadt et al, 1979; Santos, 1997). Les comportements de vacances se présentent donc ici comme un reflet de l'environnement culturel. $D^{\prime}$ autre part, une enquête montre que le sexe n'est pas un facteur discriminant dans la fréquentation du littoral français, mais avec une exception: celle de la côte d'Azur où près des deux tiers des touristes sont des femmes. Et cela sans doute à cause de la combinaison de deux facteurs: 1'importance dans cette région des séjours en résidence secondaire, chez des parents ou des amis, synonyme de sécurité pour les femmes et importance des séjours effectués par des personnes inactives, soit de 25 à 44 ans (mères de famille) soit de plus de 55 ans (retraitées) (Ministère de l'Equipement, des Transports et du Tourisme, 1993). Ainsi apparaît une diversité entre les femmes (Le Feuvre, 1994) même si on constate des lignes de force influençant globalement leur loisir, ce dernier fait étant souvent attribué à leur position dans une société patriarcale (Dawson, 1988).

\section{CONTRAINTES PESANT SUR LE LOISIR DES FEMMES ET SUR LEURS VACANCES}

Certaines des contraintes pesant sur leur loisir sont objectives (limitations temporelles, économiques, socio-démographiques, difficultés d'accessibilité, manque de moyens de transport) et peuvent aussi affecter celui des hommes. Mais lourdes sont aussi les contraintes subjectives qui leur sont spécifiques: peur de la violence chez soi et en dehors du foyer (Deem, 1996, 116); éthique du soin qui place au premier plan les relations avec les autres, le devoir de s'occuper d'eux, souvent liế au sentiment de ne pas avoir droit au loisir (Gilligan, 1982; Deem, 1986; Shank, 1986; Green and Hebron, 1988), ce qui entraîne un état de culpabilité si on s'accorde un peu de loisir (Shank, 1986; Allison and Duncan, 1987). On peut parler ici d'une idéologie, celle du familisme (quelquefois appelée familialisme (Shaw, 1992), selon laquelle les rôles sociaux principaux de la femme sont ceux d'épouse et de mère et qui constitue pour les femmes une contrainte (Kay, 1996) à la fois objective et subjective (Harrington, Dawson et Bolla, 1992).

Il faut noter aussi une différence importante: $c^{7}$ est celle qui caractérise la relation respective des hommes et des femmes au temps (Mercure, 1988; Graham, 1984) et à l'espace (Sullerot, 1970; Mowl and Turner, 1995), relation dont 1'impact est particulièrement marqué pendant les vacances: en effet, que celles-ci impliquent ou non un départ, la relation quotidienne au temps et à l'espace se trouve alors modifiée.

Dans ce contexte, il n'est pas étonnant que les activités de loisir varient en qualité comme en quantité selon le sexe, y com- pris pendant les vacances. On a montré, à propos du loisir en général, que les femmes préfèrent les activités plus expressives et plus sédentaires (Deem, 1986) et donnent plus d'importance aux relations et obligations sociales au cours de leur loisir (Deem, 1986; Lenskij, 1988; Bella, 1989). Celui-ci apparaît comme centré sur l'interaction sociale (Wimbush and Talbot, 1988) et c'est cette dernière qui donne son sens à leur expérience du loisir.

\section{QUELQUES ÉTUDES QUALITATIVES SUR LES VACANCES DES FEMMES}

Il existe quelques études qualitatives sur les vacances des femmes mais elles sont peu nombreuses. Quelques-unes ont concerné les vacances familiales dont elles ont souligné le côté ambivalent, montrant notamment que les voyages, s'ils peuvent être source d'échanges positifs entre les différents membres de la famille, sont susceptibles aussi de provoquer des frustrations et des conflits, par exemple à propos de la division du travail entre les sexes lorsque se présentent des tâches. inhabituelles pendant la vie quotidienne (Rosenblatt et Russell, 1975). De même, une étude de 1984 fait apparaître que le camping peut apporter plus de tension que de détente lorsque le lieu de vacances est moins bien équipé que le domicile habituel pour les tâches ménagères (Cerullo et Ewen, 1984). Entre temps, l'analyse de la vie dans un village de vacances de la côte sud de la France a montré que la socialisation du travail ménager (c'est-àdire qu’aucun travail de ce type n"était demandé aux vacanciers pour assurer la vie collective) n' induisait pas mécaniquement l'enrichissement de l'expérience que les femmes ont des vacances, leur soumission à la famille trouvant le plus souvent d'autres relais que le travail ménager (Morville-Descolonges, 1978).

Plus tard, on a montré que la différence de sexe influence l'interprétation des sites par les touristes et les associations symboliques qu'ils font avec les endroits visités. C'est ainsi que Squire (1994) a observé et interrogé les visiteurs de la propriété de Beatrix Potter dans la région des lacs en Angleterre. Les touristes de sexe féminin associaient leur intérêt pour cette dernière, célèbre auteur de «Peter Rabbit» et autres contes pour enfants, à certaines attitudes et à certaines valeurs. 
liées à leurs souvenirs d'enfance et à leur vie de famille, le tout sous-tendu par l'idée souvent inexprimée que les femmes sont les gardiennes essentielles de la famille et des traditions. L'influence du sexe est ainsi démontrée sur la signification donnée aux lieux touristiques, les attentes et les expériences des femmes étant ici différentes de celles des hommes.

Dans son étude de Milton Keynes, au début des années 80, Deem (1996a) avait observé - vingt-six ans après Evelyne Sullerot - que les vacances étaient souvent pour les femmes un rêve décevant: c'est-à-dire qu'il y avait un décalage entre ce que les femmes observées attendaient des vacances et leur expérience vécue. Le type d'hébergement, souvent le camping/ caravaning, impliquait une continuation du travail domestique habituel, mais de plus, quel que soit ce type d'hébergement, les activités de loisir souhaitées (marche, danse, visites touristiques, plage) étaient pratiquées moins souvent qu'il n'avait été espéré. Non seulement à cause de la nécessité de répondre aux désirs des enfants, mais aussi à cause de conflits dans les choix avec les époux ou compagnons. La routine des vacances n'était pas perçue comme aussi différente de celle de la vie habituelle qu'on aurait pu le penser. L'auteure indique que certaines mères de famille revenaient même de leurs vacances aussi fatiguées et stressées qu'à leur départ et qu'elles considéraient souvent le rythme des vacances comme survolté. Pour les femmes célibataires sans parents dépendants et sans partenaire masculin, les vacances étaient ressenties comme plus détendues et plus proches de leurs attentes, leur permettant d'échapper à la routine et au rythme trop rapide de leur vie quotidienne. Pour elles existait le sentiment d'avoir en vacances un temps à soi contrastant avec la rigueur du temps de l'horloge (Deem, 1996a: 18).

Poursuivant l'étude des vacances des femmes, Deem et ses collègues Penny Tinkler et Wendy Langford ont mené une enquête à Lancaster en 1994. Leur échantillon présente l'intérêt particulier d'être composé non seulement de touristes mais aussi de femmes résidant dans la ville et y passant leurs vacances. Par ailleurs, c'est un échantillon présentant une gamme très variée à propos de l'âge et du statut familial. Parmi l'ensemble des répondantes. un peu plus de $40 \%$ avaient récemment passé des vacances à la maison mais sans

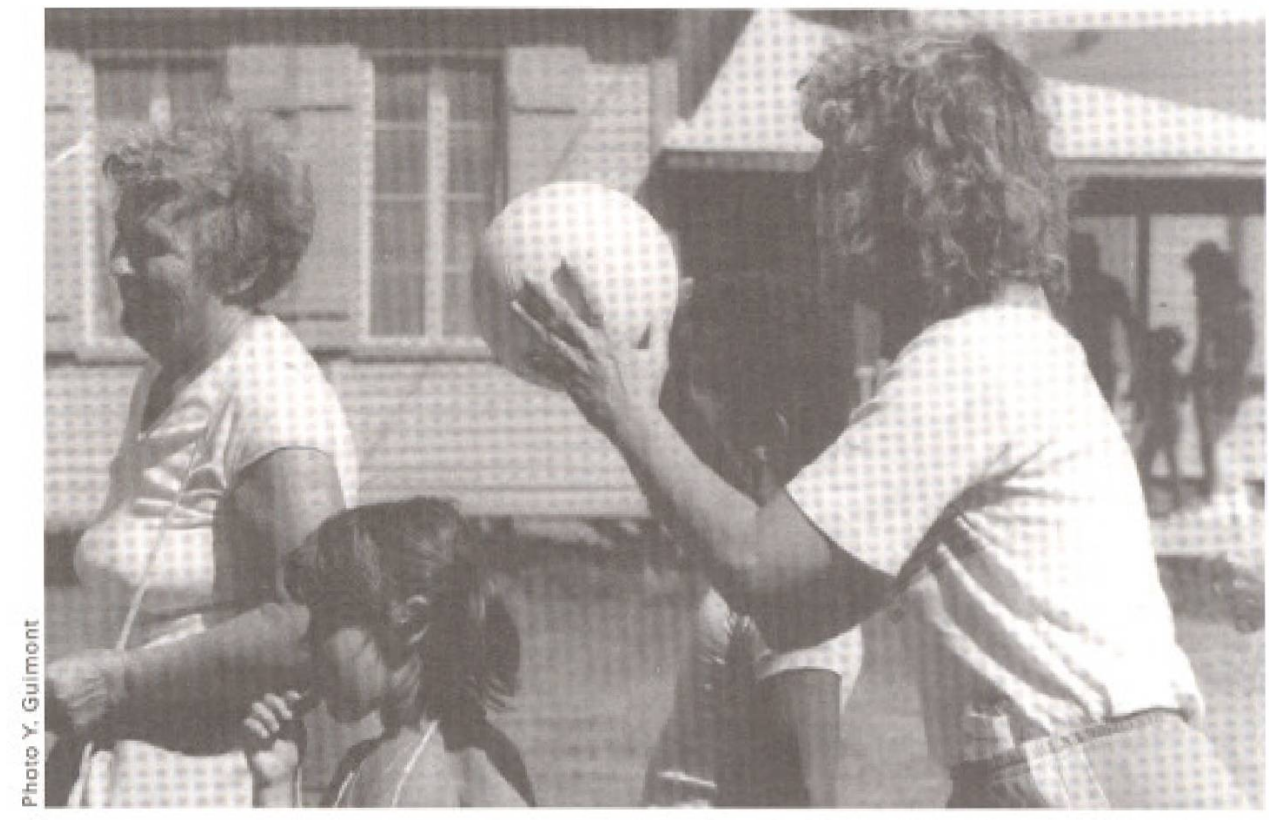

respecter la routine habituelle. Dans la totalité du groupe, certaines étaient responsables d'autres personnes; certaines ne l'étaient pas. L'enquête montre que le temps de vacances passé à la maison était ressenti comme qualitativement différent de celui des soirées et des week-ends habituels: il était décrit comme plus flexible, moins intense et moins soumis à la demande des autres. Pour les femmes ayant un partenaire masculin et/ou des enfants, le travail domestique et le soin des enfants persistaient mais c'était souvent aussi le cas pour les femmes parties en vacances. Bien entendu, comme le souligne Deem (1996a: 20-21), différents facteurs comme la classe sociale, le capital culturel, l'histoire personnelle sur le plan du travail et de l'identité jouent ici un rôle que des recherches futures devraient préciser.

Quant aux vacances des mères de jeunes enfants, elles ont été étudiées en Australie au moyen d'entretiens semi-directifs auprès d'un échantillon de femmes disposant des niveaux de revenus les plus variés (Davidson, 1996). La plupart $\mathrm{n}^{*}$ avait pas d'emploi mais certaines travaillaient à plein temps et d'autres à temps partiel. L'auteure souligne la diversité de leur expérience: pour certaines, il n'y a de vacances que si les obligations familiales disparaissent; d'autres apprécient leur repos relatif dans le cadre familial et le vécu d'un temps qualitativement positif en tant que mère et compagne. Pour toutes, les caractéristiques principales des vacances sont la réduction de la pression habituelle et la possibilité de maintenir et de développer des relations sociales (Davidson, 1996: 92). L'allégement de la pression peut exister sans quitter le domicile; cela peut être le cas quand ce sont les enfants et le compagnon qui partent ou quand quelqu'un vient à la maison pour partager la charge de travail. Toutes les répondantes indiquent que les vacances en famille exigent moins de travail, moins d'efforts que la routine normale. Qu'elles restent à la maison ou qu'elles partent, elles définissent les périodes de moindre pression comme des vacances, celles-ci n'étant pas ressenties comme une absence de pression mais comme une diminution de la tension habituelle. S'il n'y a pas de réduction de pression, il n'y a pas de vacances. Enfin l'étude souligne l'importance des relations personnelles pendant les vacances des femmes, à l'intérieur ou à l'extérieur de la famille. Il s'agit là souvent du développement, voire de la reconstruction, de l'identité et de la personnalité, soit par le raffermissement du rỏle familial souvent effacé dans la vic quotidienne soit par l'épanouissement individuel dans un temps à soi.

Mais dans l'état actuel des connaissances, cette dernière éventualité ne semble jouer que pour un petit nombre de femmes. Pour la majorité, on pourrait conclure par cette brève expression: pas de miracle! Pour le plus grand nombre en effet, les vacances $\mathrm{n}^{+}$apparaissent pas comme un temps de loisir orienté vers la réalisation de la personne comme fin dernière ct apportant bien être, relaxation, détente, plaisir, mais au mieux comme un temps ne comportant qu'à un certain degré ces caractéristiques, 
un temps où sont trop souvent reproduites les inégalités et les contraintes qui pèsent sur la vie habituelle des femmes. Il faut noter que la plupart des rares recherches sur leurs vacances concernent les mères de famille. Des études sont souhaitables sur les vacances des autres catégories de femmes, y compris la minorité des privilégiées qui descendent à l'hôtel ou partent en croisière! Mais peut-être faudrait-il mener en particulier des études longitudinales sur les vacances des petites filles et des adolescentes et sur le rỏle de ces vacances dans la création de leurs attentes et de leurs comportements non seulement lors de leurs vacances à l'äge adulte mais aussi dans l'ensemble de leur existence.

Un comité de lecture a lu et accepté ce texte

\section{BIBLIOGRAPHIE}

Allison, M. et M. Duncan (1987), sWomen, Work and Leisure: The Days of our Livess, Leisure Studies, no 9, p. 143-169.

Bella, L. (1989), women and Leisure: Beyond Androcentrism*, dans E. Jackson et T. Burton (dir.). Understanding Leisure and RecreationMapping the Past, Charting the Future, State College, Pennylvania, Venture, p. 151-180.

Cerullo, M. et P. Ewen (1984), wThe American Family goes Camping: Gender, Family and the Polities of Spaces, Antipode, no 16, p. 35-45.

Communauté Européenne (1986), Les departs en wacances des Européens: Enquête réalisée par sondage auprès d'un échantillon représentatif de la population adulte ( 15 ans et plus) des douze pays de la communate europerene, Bruxelles.

Crawford, J., S. Kippax, J. Onyx, U. Gault et P. Benton (1992), Emolion and Gender: Constructing Meaning from-Memory, London, Sage.

Davidson, P. (1996), aThe Holiday and Work Experience of Women with Young Childrens, Leisure Studies, no 2, April, p. 89-103.

Dawson, D. (1988), *The Rational Subordination of Women's Leisure under Patriarchal Capitalisms, Loisir et Société, 11, no 2, p. 397411.

Deem, R. (1986), All Work and No Play? The Sociology of Women and Leisure, Milton Keynes: Open University Press.

(1992), *The Sociology of Gender and Leisure in Britain. Past Progress and Future Prospects\%, Loisir et Sociéte, 15, no 1, p. 21-38. (1996a), *No Time for a Rest? An Exploration of Women"s Work, Engendered Leisure and Holidays., Time and Society, 5, no 1, p. 5-25.

(1996b), Women, the City and Holidaysw, Leisure Studies, no 2, April, p. 105-119.

Dumazedier, J. (1974), Sociologie empirique du loisir, Paris. Le Seuil.

England, P. M.S. Herbert, B. Stanek Kilbourne, L.R. Reid et L. Mc Creary Magdal (1994), whe Gendered Valuation of Occupations and Skills: Earnings in 1980 Census Oceupationsw, Social Forces, 73, p. 65-99.

Gilligan, C. (1992), In a Different Voice, Cambridge, Ma, Harvard University Press.

Graham. H. (1984), Women, Health and the Family, Brighton, Harvester Press.

Green, E. et S. Hebron (1988), weisure and Male Partnersw, dans E. Wimbush et M. Talbot (dir.) Relative Freedoms. Milton Keynes, Open Uni= versity Press.

Harrington, M. D. Dawson et P. Bolla (1992). *Objective and Subjective Constraints on Women's Leisurem, Loisir et Societe, 15, no 1. p. 203-222.

Henderson, K.A. (1991), \&The Leisure Gap: Constraints to Leisure for Womens, WLRA Congress on Leisure and Tourism, Sydney, Australia, University of Technology.

Kadt, E. et al. (1979), Tourisme, Passeport pour le développement? Paris, UNESCO et Banque mondiale, p. 64.

Kay, T. (1996), women's Leisure and the Family in Contemporary Britain*, dans N. Samuel (dir-) Women, Leisure and the Family in Contemporary Society A Mutinational Perspective, Wallingford. CAB International.

Lanquar. R. (1994). Sociologie du tourisme et des woyages, Paris, P.U.F., Collection Que saisje? no 2213 .

Lanquar, R. et Y. Raynouard (1995), Le towrisme social et associatif, Paris. P.U.F. Collection Que sais-je? no 1725 .

Le Feuvre, N. (1994), sLeisure, Work and Gender: A Sociological Study of Women's Time in Frances, Tume and Soclety, 3, no 2, p. 151-178.

Lenskij, H. (1988), alMeasured Time: Women, Sport and Leisure*, Leisure Studies. 7, p.233240 .

Mercure, D. (1988), sL'étude des temporalités chez les femmes. Une remise en question des catégories usuelles d'analyses, dans D. Mercure et A. Wallemacq (dir.), Les Temps sociaux, Bruxelles, De Boecq Westmael.

Ministère de l'Equipement, du Logement, des Transports et du Tourisme (1997), Memento du Tourisme, Paris, Direction du Tourisme.
Morville-Descolonges, M. (1978), "A propos de la socialisation du travail domestique: I'analyse d'un village de vacances", Imternational Journal of Urban and Regional Research, 2, no 3, p. 482-498.

Mowl, G. et J. Towner (1995), «Women, Leisure and Place: towards a more "humanistic" Geography of Women's Leisures, Leisure Studies, 14, p. 102-106.

Rauch, A. (1993), Les vacances, Paris, PUF, Collection Que sais-je?, no 2749 .

Richez, J.C, et L. Strauss (1995), «Un temps nouveau pour les ouvriers: les congés payés (1930-1960)\%, dans A. Corbin (dir), L'Avènement des loisirs 1850-1960, Paris, Aubier, p. 376412.

Rosenblatt, P.C. et M. Russell (1975), w The Social Psychology of Potential Problems in Family Vacation Travels, The Family Coordinator, 24, no 2, April, p. 209-215.

Roy. C. (1990), wes Emplois du temps dans quelques pays occidentaux*, Données Sociales, Paris, INSEE, p. 223-225.

Samuel. N. (dir.) (1993), L'Evolution historique du tourisme de masse en Frances, dans C. Condominas (dir, ), Les Loisirs au Japon. Paris; L'Harmattan, Collection Logiques Sociales, p. 205-221.

(1996), Women, Leisure and the Family in Contemporary Society: a Multinational Perspective. Wallingford, CAB International.

Santos, A. (1997) «Interrail: The Train Odisseys, mémoire de maîtrise, WICE, Wageningen, PaysBas.

Shank, J. W. (1986), «An Exploration of Leisure in the lives of Dual-Career Womens, Journal of Leisure Research, 18, p. 300-319.

Shaw, S, (1992), wDereifying Family Leisure: an Examination of Women's and Men's Everyday Experiences and Perception of Family Times, Leisure Sciences, 14, p. 271-286.

Sondage SOFRES (1995), Les vacances des Français (échantillon représentatif de 6000 personnes), Paris.

Squire, S.J. (1994), aGender and Tourist Experiences: Assessing Women "s Shared Meanings for Beatrix Pottere, Leisure Studies, 13, 195 209.

Sullerot, Evelyne (1970), La femme et les loisirs, Paris, Etats Généraux de la Femme, 12 novembre.

United Nations (1991), World's Women, 1979 . 1990: Trends and Statistics, New York.

Wimbush, E. et M. Talbot (dir.) (1988), Relative Freedoms, Milton Keynes, Open University Press. 